\title{
Optimization of cultural conditions for in vitro regeneration of wild potato species
}

Koloshina K.A. ${ }^{1 *}$, Egorova A.A. ${ }^{1}$, Chalaya N.A. ${ }^{2}$, Rogozina E.V. ${ }^{2}$, Gerasimova S.V. ${ }^{1}$

${ }^{1}$ Institute of Cytology and Genetics, SB RAS, Novosibirsk, Russia

${ }^{2}$ N.I. Vavilov All-Russian Institute of Plant Genetic Resources, St. Petersburg, Russia

*email: kristina.koloshina@yandex.ru

The wild tuberous Solanum species growing in the countries of South America are a valuable donor material for cultivated potato breeding. A number of experimental approaches in wild potato biotechnology require the step of in vitro regeneration. The aim of this study was to evaluate the in vitro regeneration process of wild potato species on various culture media. The work involved 5 different protocols for callus induction (CIM) and regeneration (RM) nutrient media, and 14 wild potato samples belonging to different species from VIR Genebank collection. The stem explants were incubated at CIM until calli were formed, and then transferred to RM. The number of explants producing regenerated plants was counted. Callus formation was observed in 13 species, among them formation of regenerated plants was noted in 11 species. The samples that formed the largest number of regenerating plants belong to S.pinnatisectum, $S$. chacoence and $S$. polyadenium species. One of the experimental media was found to be suitable for the regeneration of all 11 species. This medium can further be used for different biotechnological methods, for example, for genetically modified plants regeneration after genetic transformation of potato cells.

Acknowledgements: The work was carried out with the financial support of the RFBR grant No. 20-016-00217. 